\title{
Recreational genomics? Dreams and fears on genetic susceptibility screening
}

\author{
European Journal of Human Genetics (2008) 16, 403-404; doi:10.1038/ejhg.2008.32
}

In the previous century genetics in health care had a strong focus on monogenic and chromosomal disorders. Clinical genetics developed in several countries as a medical specialty, aiming to assist individuals and families to cope with a diagnosis of a severe genetic health problem. Complex decisions on reproductive issues were supported by information and clarification. Protocols were developed to enable people to make informed decisions on choices to test or not to test for disorders with a high impact such as Huntingtons disease and hereditary cancer syndromes.

However, in this century, developments in genetics and genomics have taken a different course. Complex diseases are being studied, sometimes with monogenic subsets but mainly with multifactorial etiology. What used to be a geneticists nightmare was the success of the year 2007: the unravelling of the genetic basis of type II diabetes at a high pace. ${ }^{1}$ Valorization into genetic tests directly available to consumers through the internet, followed rapidly. Are your personal risk profile and tailored prevention advice now for sale? Has science progressed in a never precedented way, or are we witnessing a hype of naive and commercial hopes and beliefs?

Criteria for screening in the low-risk population were developed as early as 1968 by the World Health Organization. $^{2}$ These so-called Wilson and Jungner ${ }^{2}$ criteria mention several aspects to balance pros and cons of screening possibilities, and to evaluate whether benefits outweigh disadvantages. A central criterion was the availability of treatment. Genetic screening has widened the concept of treatment to include other options that users of health care might consider useful, namely reproductive options, advice on prevention or 'just' the possibility to be better prepared for the life to come. Several sets of criteria have been developed, based on the Wilson and Jungner ${ }^{2}$ framework. Further development included for instance the quantitative evaluation of what could be considered a 'suitable' screening test: sensitivity, specificity, positive predictive value, number of false positives, analytic validity and clinical validity. ${ }^{3,4}$ Recently developed assessment schemes focus on 'clinical utility' which in short reflects whether people will become more healthy or happy, will live longer and in better health, based on the result of a screening test and the options available for people at a high risk. A diabetes risk test based on TCF7L2 genotype may now be available, and the advice to keep your BMI between 20 and 25 , to take care of physical activity at least $30 \mathrm{~min}$ per day and to use a healthy diet may be given to the highrisk group; however, there is no evidence so far that this advice is effective. Reducing weight and increasing physical activity is effective in reducing cardiovascular morbidity and mortality risks, but this does not imply that an advice to do so is effective. A recently published randomised controlled trial among children of type II diabetes patients showed no significant effect of a 1-year behavioral change program delivered at home or by telephone, in decreasing weight or increasing physical activity. ${ }^{5}$

It looks like we are getting more and more confident about some genetic associations, and can estimate individual risks of complex diseases more precisely that a year ago. Although the average inhabitant of Netherlands now has a lifetime risk of developing type II diabetes of $13 \%$, for some people this might be 10 or $17 \%$ after testing. Whether this makes any difference to people is not known. Effective interventions to reduce a risk of $17 \%$ to the population average of $13 \%$ are not yet available. Whether paying US $\$ 300$ or even $\$ 1000$ helps to motivate people to follow-up their individual lifestyle advice is not known either.

Until recently neither the genetics community, nor any other specialty communicated much about the genetic aspects of type II diabetes. ${ }^{6}$ Now commercial companies succeed in getting the message to the market, so the future may tell how useful this is.

The Public and Professional Policy Committee (PPPC) of the European Society for Human Genetics co-organized a workshop on susceptibility screening with EUROGENTEST and with the Institute for Prospective Technological Studies in Seville, Spain, in October last year. Background documents and recommendations that were produced by PPPC in the past are available on www.eshg.org. EJHG will 
publish in the year 2008 a background document and recommendations on patenting. For these documents consultation rounds have been finished. In the year 2008, the ESHG website will be used for consultation on pharmacogenomics, genetic susceptibility screening, testing of minors and direct-to-consumer testing. The PPPC welcomes your comments and encourages national genetics organizations as well as health-care professionals and policy makers to use the finalized documents to improve debate and guide policy, as well as to respond to the drafts of documents.

During the Seville meeting in October 2007, geneticists recognized the challenges related to susceptibility testing. Too many questions remain unanswered today. Education and communication are needed. Cost-effectiveness often has not been studied. How can new genetic tests be properly evaluated before getting Conformité Européenne marking? How can physicians be prepared now that they can expect clients entering their consulting room with a high-risk estimate provided by a genetic company and based on a whole genome scan $?^{7}$ A New England Journal of Medicine editorial of January 10, 2008 warns premature marketing of genetic tests and even coins the term 'recreational genomics'. ${ }^{7}$ Translational medicine demands more than an advertisement and an information leaflet. Multifaceted evaluation of genetic tests for clinical applications will be a major effort, not only focusing on the test properties but also on the clinical utility. ${ }^{7}$

Genetic knowledge relevant for daily practice was insufficient in many health-care workers just a few years ago, before the advent of the genome-wide association studies. ${ }^{8}$ A detailed consumer report of this genome map will almost certainly be beyond the current physicians skill sets. $^{7}$

Europe is not America. Should we consider the NEJM warnings irrelevant for Europe? Certainly not. Because of the global market the tests available direct-to-consumer can be ordered from almost anywhere. But we should also reflect on some changes in health-care systems that have progressed less in Europe than they have in the United States, and that relate to the increasing promises of individualized medicine driven by the advances in genomics. ${ }^{9}$ Health-care systems in Europe are slowly following the American example of a market-oriented system with increasing individual responsibility. However, some argue that genomic medicine asks for universal coverage. ${ }^{9}$ The discovery of individually increased disease risks typically raises health-care costs at short term, and has advantages at long term. Clients tend to change medical insurance company within 5-10 years, so insurers have little motive to engage in long-term preventive care. Genomic health care has the potential to reduce aggregate cost of health care by enabling better preventive strategies, but calls for a health-care system that is not fragmented. The emergence of individualized medicine is a compelling reason to deliver universal health care. ${ }^{9}$ For once, in the field of individualized health care, the European lack of progress may become our strength indeed.

\section{References}

1 Frayling TM, McCarthy MI: Genetic studies of diabetes following the advent of the genome-wide association study: where do we go from here? Diabetologia 2007; 50: 2229-2233.

2 Wilson JMG, Jungner G: Principles and Practice of Screening for Disease. Geneva: World Health Organization, 1968.

3 Janssens ACJW, Carolina Pardo M, Steyerberg EW, van Duijn CM: Revisiting the clinical validity of multiplex genetic testing in complex diseases. Am J Hum Genet 2004; 74: 585-588.

4 Janssens ACJW, Khoury MJ: Predictive value of testing for multiple genetic variants in multifactorial diseases: implications for the discourse on ethical, legal and social issues. It J Publ Health 2006; 3: $35-41$.

5 Kinmonth AL, Wareham NJ, Hardeman W et al: Efficacy of a theory-based behavioural intervention to increase physical activity in an at-risk group in primary care (ProActive UK): a randomised trial. Lancet 2008; 371: 41-48.

6 van Esch SCM, Cornel MC, Snoek FJ: Type 2 diabetes and inheritance: what information do diabetes organizations provide on the Internet? Diabet Med 2006; 23: 1233-1238.

7 Hunter DJ, Khoury MJ, Drazen JM: Letting the genome out of the bottle-will we get our wish? N Engl J Med 2008; 358: 105-107.

8 Baars MJH, Scherpbier AJJA, Schuwirth LW et al: Deficient knowledge of genetics relevant for daily practice among medical students nearing graduation. Genet Med 2005; 7: 295-301.

9 Evans JP: Health care in the age of genetic medicine. JAMA 2007; 298: $2670-2672$.

GertJan B van Ommen ${ }^{1}$ and Martina C Cornel ${ }^{2}$ ${ }^{1}$ Department of Human Genetics, Center for Human and Clinical Genetics, Leiden University Medical Center, Leiden, The Netherlands; ${ }^{2}$ Department of Clinical Genetics, VU University Medical Centre, Amsterdam, The Netherlands 